\title{
Missouri workshop on methods for life history data analysis
}

\author{
John D. Kalbfleisch • Jianguo Sun
}

Published online: 16 February 2010

(C) Springer Science+Business Media, LLC 2010

This special issue features contributions from seven invited speakers who participated in the Workshop on Methods for Life History Data Analysis held at the University of Missouri, Columbia, October 16-18, 2008. The workshop is part of a series of conferences on applied statistics that was previously known (from 2000 to 2004) as The Winemiller Symposium on Applied Statistics. The goal of these symposia is to communicate the value of statistical and methodological techniques in an applied setting. Originally, the Winemiller Symposium was designed to help create a campus-wide community of scholars at the University of Missouri, Columbia. To serve this purpose, each year the symposium presented two internationally known and prominent keynote speakers (one from the statistical community and one from another field such as psychology, human genetics or political science). In addition, the Symposium featured contributed presentations from scholars from the University of Missouri who were using statistics in a wide variety of disciplines. In 2004, the Symposium Committee decided to redefine the symposium as a national conference to be held every two years, each time on a new and important applied topic. This was the third conference in this new format.

Life history data arise in many fields including medicine, public health, demography, economics, finance, engineering, political science, psychology and sociology. One important type of life history data is failure time data, which corresponds to studies on the time until the occurrence of a certain event such as death, HIV infection and cancer tumor onset. An extensive literature has been developed for failure time

\footnotetext{
J. D. Kalbfleisch

University of Michigan, Ann Arbor, USA

e-mail: jdkalbfl@umich.edu

J. Sun $(\bowtie)$

University of Missouri, Columbia, USA

e-mail: sunj@missouri.edu
} 
data analysis, but there are still many papers in this area and a wealth of questions that have not been fully discussed. But the full field is much broader, including various complex outcomes that may involve series of recurrent events or multistate models of various life events that unfold in time. Many different sampling schemes are combined with these myriad structures to produce a rich and vital area of research. The Missouri workshop had sixteen invited speakers who included some of the top experts and researchers in life history data analysis; the goal of the workshop was to provide a forum for discussing issues and sharing ideas about new developments in life history data analysis.

In the first paper, Griffin and Lagakos discuss nonparametric estimation of the distribution functions of sojourn times in a finite state disease progression model in the presence of interval censoring. An algorithm is developed and the uniqueness of the estimates discussed. There are three other papers concerned with the analysis of univariate failure time data. Motivated by a problem in genomic studies, Huang and Ma consider a variable selection problem for the accelerated failure time model with high-dimensional covariates. They develop an efficient iterative algorithm and examine its properties. Lee and Whitemore investigated the relationship between proportional hazards model regression and models obtained from threshold criteria. They show that in most situations, the former model can be regarded as a special case of the latter. Finally, McKeown and Jewell discuss the analysis of current status data when the observations on current status information are subject to error.

Multivariate failure time data also occur in many studies and were the subject of four articles included in this volume. Zhang, Zhang, Chaloner and Stapleton propose a copula model for bivariate failure time data and apply it to the Multicenter AIDS Cohort Study. Recurrent event and longitudinal data are also common types of life history data. Motivated by a study on the effect of vitamin A supplements on diarrhea morbidity and severity, Cai, Zeng and Pan propose a marginal rate model for a recurrent event process and a proportional mean model for a related marker process. Estimating equations form the basis of the statistical analysis. Sun investigates a proportional mean model for general longitudinal data and develops a novel sampling adjusted estimation procedure. The new procedure is more robust to model misspecification and more efficient than the existing estimation procedures.

Professor Steve Lagakos co-authored the lead article in this special issue, and we note with sadness that this is being published posthumously. Steve was a giant in our field who made numerous contributions to research and education in biostatistics and to many areas of the medical sciences, most recently in the area of AIDS research. Each of us was fortunate to have had Steve as a colleague or mentor in the course of our careers and, like many in our discipline and elsewhere, we have benefited from his insights, intellectual honesty and generosity of spirit. Biostatistics has been very much the richer for him and we shall all miss him greatly.

It has been our privilege to co-edit this special issue. We would like to thank the Lifetime Data Analysis Editor-in-Chief, Professor Mei-Ling Lee, for inviting us to assemble this special issue. Likewise, we are indebted to colleagues who helped review the articles and to Ms. Gayathri Balasubramanian, the journal's editorial staff, for her assistance and particularly for her patience as we tried, with varying degrees of success, to navigate the Journal's web-based editorial system. 